Annuaire suisse de politique de développement

4 | 1984

Annuaire Suisse - Tiers Monde 1984

\title{
Le nouveau crédit-programme de la Coopération technique et de l'aide humanitaire : un message a la fois accablant et encourageant
}

\section{Gilbert Coutau}

\section{OpenEdition}

\section{Journals}

Édition électronique

URL : http://journals.openedition.org/aspd/1165

DOI : 10.4000/aspd. 1165

ISSN : 1663-9669

Éditeur

Institut de hautes études internationales et du développement

\section{Édition imprimée}

Date de publication : 1 janvier 1984

Pagination : 143-146

ISSN : $1660-5934$

\section{Référence électronique}

Gilbert Coutau, « Le nouveau crédit-programme de la Coopération technique et de l'aide humanitaire un message a la fois accablant et encourageant », Annuaire suisse de politique de développement [En ligne], 4 | 1984, mis en ligne le 02 février 2013, consulté le 08 septembre 2020. URL : http:// journals.openedition.org/aspd/1165; DOI : https://doi.org/10.4000/aspd.1165 
I.

Le nouveau crédit-programme de la coopération technique et de l'aide humanitaire :

UN MESSAGE A LA FOIS ACCABLANT ET ENCOURAGEANT

Gilbert Coutau

Le message du Conseil fédéral est un document de grande valeur informative et d'un poids politique incontestable. II n'est pas seulement l'occasion de faire le point sur la conception générale que la Suisse se fait dans ce domaine. II résume succinctement la situation où se trouvent ces pays après une trentaine d'années de "politique de développement" menée au niveau international et national.

Tout en rappelant les diverses articulations de l'aide publique suisse au développement, il évoque plus particulièrement les résultats concrets obtenus dans le cadre du crédit de programme adopté en décembre 1980.

L'analyse est réaliste car elle ne dissimule ni les ambiguités, ni les obstacles, ni les limites. Mais elle peut aussi se référer avec satisfaction à l'appréciation du CAD qui juge la politique suisse du développement, sinon suffisante du point de vue quantitatif, du moins particulièrement efficace du point de vue qualitatif. Enfin, le message situe bien les priorités que le Conseil fédéral s'est fixées dans I'utilisation du nouveau crédit de programme de 1.800 millions de francs portant au minimum sur trois ans.

A maints égards, le document du Conseil fédéral est à la fois accablant et encourageant. Accablant car, devant l'ampleur de la tâche, l'étendue de la diversité des problèmes, on peut douter de l'apport réel de la contribution suisse. Qu'il s'agisse des catastrophes naturelles, climatiques et écologiques, des effets des évolutions démographiques incontrôlées (auxquelles on semble enfin prêter une attention plus soutenue), y compris les migrations spontanées ou contraintes, des séquelles particulièrement dramatiques pour les pays en voie de développement de la crise économique mondiale et du poids de leur surendettement, ou encore des instabilités sociales et politiques internes, qui pèsent sur l'affectation des ressources disponibles et de la fragilité des appareils administratifs de la plupart de ces pays qui en compromettent le bon usage, tous ces éléments et quelques autres sont de nature à décourager. A quoi bon la goutte d'eau de l'aide suisse dans l'aridité et l'immensité de ce catalogue? 
D'un autre côté, il y a aussi de bonnes raisons de persévérer. D'abord, le message établit les distinctions indispensables et trop souvent négligées entre les divers pays en développement. Dans plusieurs cas, les interventions conjuguées des pays industrialisés ont porté des fruits substantiels. II n'est pas toujours facile de discerner la ou les clés qui ont permis de sortir du cercle vicieux du sous-développement. Le fait est néanmoins que dans des domaines comme la santé, l'éducation, l'indépendance alimentaire et les taux de croissance des échanges, des progrès très réels ont permis à des groupes de pays de devenir de véritables partenaires sur la scène économique mondiale, pour le plus grand bien de leur population.

\section{Rôle des investissements privés}

On souligne avec intérêt à ce sujet les considérations beaucoup plus positives que les investissements directs privés inspirent aux auteurs du message. Insistant sur l'importance du "climat d'accueil" réservé à ces transferts privés, le Conseil fédéral relève en particulier le rôle de stimulation qu'ils jouent à maints égards. A juste titre, il ajoute que des règles plus claires devraient être établies quant aux conditions de transfert et au comportement des entreprises transnationales. Mais le gouvernement entend que le crédit de programme contribue à financer des mesures destinées à favoriser l'engagement de ressources du secteur privé dans la réalisation de projets industriels. En revanche, pour les pays les plus pauvres, c'est bien l'aide publique qui reste prioritaire, conformément d'ailleurs à la pratique suisse.

Cette déclaration est d'autant plus importante que, selon I'OCDE, "si I'on ajoute les apports totaux privés et publics de la Suisse, on obtient avec 3,2\% du PNB le chiffre le plus élevé parmi les pays du CAD".

\section{Stimuler "l'effort propre" des destinataires}

Parmi les nombreuses et pertinentes observations contenues dans ce document, j'aimerais faire ressortir encore deux éléments auxquels j'accorde une importance particulière. II s'agit d'abord de l'accent mis sur l'amélioration nécessaire de ce que l'on pourrait appeler la capacité de réception que les pays en développement peuvent manifester à l'égard de l'aide étrangère. Plusieurs chapitres sont consacrés en effet à l'encouragement de "I'effort propre" de ces pays.

En arrière-plan, on évoque la problématique classique liée à l'aide alimentaire. Ces passages méritent d'être soulignés. En voici un parmi d'autres: "Aussi importantes soient-elles, l'action de l'Etat et celle des agences d'aide ne feront que brasser le vide ou mettre à leur actif des réalisations sans lendemain si elles ne peuvent s'appuyer sur cet effort individuel et collectif qui témoigne de la volonté des intéressés de ne pas plier devant la misère et la prodigieuse force d'inertie qui s'en dégage."

Ecrire que "la responsabilité première du développement incombe aux pays 
en développement eux-mêmes" n'est pas un sacrilège. C'est une réalité qui doit inspirer une partie importante des priorités dans le choix des programmes. Mais c'est aussi affirmer le respect de la souveraineté des autorités locales dans I'orientation et la réalisation de l'aide. II n'y a pas seulement de la modestie, mais un grand réalisme à déclarer que "cette option implique en premier lieu que l'on renonce à imposer des solutions de l'extérieur, quand bien même elles auraient fait leurs preuves sous d'autres latitudes, et que l'on recherche la zone de convergence de la stratégie de développement choisie par le pays récipiendaire et des objectifs de notre politique d'aide tels que les définit la loi du 19 mars 1976.

Elle exige, en second lieu, une profonde connaissance du pays bénéficiaire, de ses institutions et des forces vives de la société, à l'échelon national et local, le choix du partenaire étant aussi important que celui des activités envisagées. Enfin, cette option appelle, particulièrement dans le cas d'un soutien aux initiatives de petites collectivités locales, une méthodologie faite de prudence et de patience. Ne pas fixer d'objectifs qui n'aient été agréés par les destinataires; ne pas imprimer à la marche du projet un rythme plus rapide que celui que veulent suivre ses bénéficiaires; ne pas enfermer le déroulement du projet, sous prétexte de planification, dans un carcan qui I'empêche de réagir à la dynamique qu'il aura lui-même contribué à déclencher."

Dans ce même contexte de pragmatisme, à propos de la controverse sur la condition sine qua non du respect des droits de l'homme par les pays bénéficiaires à laquelle certains voudraient subordonner l'aide de la Suisse, le gouvernement confirme que "I'interruption d'un programme de coopération à la suite de violations flagrantes des droits de l'homme peut avoir pour conséquence d'aggraver encore les conditions de vie des populations pauvres qui ne sont pas les responsables, mais bien les victimes de la politique suivie par leurs autorités". Les situations sont donc examinées, cas par cas, sans dogmatisme pédant.

\section{Plaidoyer pour l'aide multilatérale}

Le second sujet que je voudrais relever concerne l'aide multilatérale. Cet aspect de l'aide au développement n'a pas très bonne presse, surtout auprès des institutions d'entraide qui la considèrent parfois comme une concurrence trop gourmande. Je ne partage pas ce point de vue, considérant que ces deux formes d'aide - bilatérale et multilatérale - sont par nécessité complémentaires. C'est dire que le chapitre consacré à l'action des institutions internationales mérite une attention toute spéciale à mes yeux. Le Conseil fédéral entreprend une véritable réhabilitation de l'aide multilatérale. II énumère ses avantages spécifiques comme la priorité qu'elle accorde aux pays les plus pauvres, la continuité de son action, la dimension plus étendue que ses programmes peuvent prendre, la somme d'expériences administratives et pratiques accumulées dans la planification, la surveillance et le contrôle de tra- 
vaux de vaste ampleur, le rôle de coordination entre de multiples partenaires à mettre en synergie, l'exclusivité de son potentiel d'intervention en cas de crise grave, la neutralité de l'aide offerte, qui n'est liée à aucune condition commerciale ou politique. Le gouvernement en conclut opportunément qu'un "petit pays comme le nôtre, dont l'économie est tournée vers l'extérieur, doit également pouvoir compter sur une compréhension et une coopération multilatérale au développement dans les années qui viennenț et, dans ce but, nous continuerons d'acheminer vers les institutions et programmes multilatéraux une part substantielle de l'aide publique au développement".

\section{Culpabilisation, mauvaise conseillère}

II y aurait encore à mettre en évidence beaucoup d'autres aspects de ce document. Par exemple les passages consacrés au contrôle financier et aux opérations d'évaluation des programmes projetés ou réalisés. Les problèmes d'organisation, et de structures internes de l'administration ainsi que d'intéressants aspects de collaboration avec les institutions d'entraide ou avec le secteur privé qui semble associé un peu plus organiquement que naguère à certains projets. II faut enfin saluer les quelques exemples concrets présentés sans fards dont la description illustre et explique l'approche qui se veut résolument pragmatique de l'aide publique suisse au développement.

Je suis persuadé pour ma part que ce document est de ce fait beaucoup plus convaincant que les autoflagellations et les appels à la mauvaise conscience lancés par les apôtres patentés du développement. Si l'on veut que le peuple suisse accepte une nécessaire augmentation de l'effort en faveur du développement, c'est d'abord en le présentant comme un élément majeur de notre politique de sécurité qu'on y parviendra. Le Conseil fédéral fait bien de le souligner : "La brèche croissante qui sépare riches et pauvres est source de tensions et de conflits. En contribuant à l'amenuiser, l'aide publique au développement apporte une contribution importante à la paix et à la sécurité internationales et, par là même, à la sécurité de notre pays." 